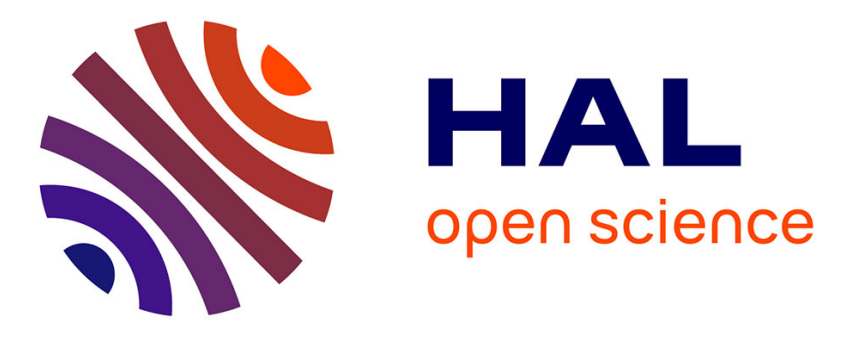

\title{
Troubles du comportement et hallucinations après consommation d'infusions de feuilles de boldo
}

\author{
Pierre-Jacques Chaboussant, Anne-Laure Gagez, Marianne Graber, \\ Jean-Michel Zhao, François Chavant, Marie-Christine Perault-Pochat, Denis \\ Graber
}

\section{To cite this version:}

Pierre-Jacques Chaboussant, Anne-Laure Gagez, Marianne Graber, Jean-Michel Zhao, François Chavant, et al.. Troubles du comportement et hallucinations après consommation d'infusions de feuilles de boldo. Thérapie, 2014, pp.Letters to editor. 10.2515/therapie/2014052 . hal-01070837

\section{HAL Id: hal-01070837 https://hal.science/hal-01070837}

Submitted on 2 Oct 2014

HAL is a multi-disciplinary open access archive for the deposit and dissemination of scientific research documents, whether they are published or not. The documents may come from teaching and research institutions in France or abroad, or from public or private research centers.
L'archive ouverte pluridisciplinaire HAL, est destinée au dépôt et à la diffusion de documents scientifiques de niveau recherche, publiés ou non, émanant des établissements d'enseignement et de recherche français ou étrangers, des laboratoires publics ou privés. 


\section{Troubles du}

\section{comportement}

et hallucinations

\section{après consommation}

d'infusions de feuilles de boldo

\author{
Pierre-Jacques Chaboussant ${ }^{1}$, Anne-Laure Gagez ${ }^{3}$, \\ Marianne Graber ${ }^{3}$, Jean-Michel Zhao ${ }^{3}$, \\ François Chavant ${ }^{2}$, Marie-Christine Perault-Pochat ${ }^{2}$ \\ et Denis Graber ${ }^{1}$
}

1 Service de pédiatrie, Hopital Saint-Louis, La Rochelle, France

2 Service de pharmacologie clinique et vigilances, Centre régional, de pharmacovigilance et de renseignement sur les médicaments, Hopital de la Milétrie, Poitiers, France

3 Laboratoire CNRS UMR 7266 «LIttoral ENvironnement et Société (LIENSs) », Université de La Rochelle, La Rochelle, France

Texte reçu le 16 janvier 2014 ; accepté le 7 avril 2014

Cas déclaré au centre régional de pharmacovigilance de Poitou-

Charentes le 11 octobre 2011

Résumé - Nous rapportons un cas de troubles du comportement avec hallucinations chez une enfant de 12 ans après consommation d'infusions de feuilles de boldo. La boldine, pourrait être responsable des troubles neuropsychiatriques transitoires présentés par la patiente. L'apparition de troubles du comportement et d'hallucinations sans cause évidente doit faire rechercher la consommation de produits de phytothérapie, notamment d'infusions («tisanes »). La consommation d'infusions à base de feuilles de boldo doit être évitée chez l'enfant.

Mots clés : enfant ; intoxication ; effet indésirable ; infusion ; boldo ; boldine ; hallucinations

\begin{abstract}
Behavioural Impairments and Hallucinations after Consumption of Boldo Leaf Infusions. We report a case of behavioural impairments with hallucinations in a twelve-year-old girl, after consumption of boldo leaf infusions. The main alkaloid of boldo, named boldine, is very likely responsible for temporary neuropsychiatric disturbances present in the patient. The emergence of behavioural problems and hallucinations without any obvious cause, should lead to search for consumption of boldo leaf infusion ("tisanes"). This consumption must be avoided in children.
\end{abstract}

Keywords: child; intoxication; adverse event; infusion; Boldo; boldine; hallucinations

Abréviations : voir en fin d'article.

\section{Introduction}

Peumus boldus (ou boldo) est un grand arbuste originaire d'Amérique du Sud (Chili, Pérou) mais qui est aussi cultivé en Europe. Les feuilles de boldo sont traditionnellement utilisées sous forme d'infusion («tisane ») pour faciliter la digestion, en cas de troubles hépatobiliaires ou de constipation.

Nous rapportons ici des troubles du comportement avec hallucinations survenus chez une fille de 12 ans après consommation d'infusions de feuilles de boldo.

\section{Observation}

Une fille de 12 ans est admise en hospitalisation pédiatrique pour accès de panique avec agitation motrice, puis prostration. A l'interrogatoire de l'entourage, on ne retrouve aucun antécédent notable en dehors d'une banale colopathie fonctionnelle avec douleurs abdominales fréquentes. Il n'y a pas de notion de traumatisme crânien, ni de prise de toxiques ou de médicaments. L'enfant est très anxieuse, regarde autour d'elle avec perplexité et évite le regard des adultes. Par moment, elle s'agite et semble terrorisée. Le discours est incohérent. Elle décrit des hallucinations visuelles (araignées, serpents).

L'enfant est apyrétique. L'examen clinique est sans particularité, notamment l'examen neurologique. Le bilan biologique estnormal. Il n'y a pas de syndrome inflammatoire. L'alcoolémie est négative. La recherche de toxiques dans les urines (barbituriques, tricycliques, benzodiazépines) est négative. L'électroencéphalogramme réalisé le lendemain de l'admission est normal.

L'évolution va être progressivement favorable avec encore quelques épisodes d'agitation et d'hallucinations. La normalisation complète du comportement de l'enfant est finalement constatée 24 heures après l'admission avec absence de réapparition des hallucinations. La patiente produira secondairement 3 dessins décrivant ses hallucinations.

La reprise de l'interrogatoire permet finalement de retrouver la consommation d'infusions de feuilles de boldo (Infusion Médiflor ${ }^{\circledR}$ ) recommandée par un pharmacien à la grand-mère, car l'enfant se plaignait de banales douleurs abdominales dans le cadre de sa colopathie fonctionnelle.

Un signalement est donc réalisé auprès du centre régional de pharmacovigilance de Poitou-Charentes le 11 octobre 2011.

Avec maintenant plus de 2 ans de recul, le comportement de l'enfant reste normal sans réapparition d'hallucinations.

A distance de l'hospitalisation, la composition des infusions de sachets de feuilles de boldo commercialisés par la marque Médiflor a été étudiée au laboratoire Littoral Environnement et Société (LIENSs) de l'Université de La Rochelle. Les «tisanes » ont été réalisées avec de l'eau pure obtenue via un système Milli $Q$ (Millipore, France). Le temps d'infusion était de 10 minutes comme recommandé par Médiflor. Il s'agissait des sachets provenant de la même 
boite que celle utilisée pour la fillette. En chromatographie en phase liquide à haute performance-ultra violet (high performance liquid chromatography-ultra violet, HPLC-UV), nous avons bien retrouvé la présence de la boldine dans l'infusion. ${ }^{[1]}$ La méthode analytique que nous avons utilisée correspondait à une séparation de chromatographie liquide en phase inversée, couplée à une détection UV à 282 et $302 \mathrm{~nm}$, selon la méthode décrite par Betts. ${ }^{[2]}$ Grâce à un standard commercial de la boldine (Fluka), nous avons pu ainsi déterminer que la patiente avait consommé $1,16 \mathrm{mg}$ de boldine (1 infusion de 2 sachets de feuilles de boldo) tous les soirs pendant 3 jours soit une dose quotidienne de $26 \mu \mathrm{g} / \mathrm{kg}$. Une confirmation plus précise de la molécule a été réalisée par chromatographie en phase liquide à très haute performance (ultra high performance liquid chromatography, UHPLC) couplée à la spectrométrie de masse, en mode positif selon une méthode mise au point au laboratoire LIENSs. En effet, le temps de rétention (1,67 min) associéà la masse exacte $(\mathrm{m} / \mathrm{z}, 328,1547 \pm 0,6 \mathrm{ppm})$ de la boldine protonée $\left(M+H^{+}\right)$ permet d'affirmer sa présence. Le matériel utilisé était un XEVO G2-S QTof couplé à une UPLC Acquity-H (Waters, Milford, EtatsUnis). La méthode UHPLC consistait à utiliser une colonne Acquity UPLC BEH C $18(2,1 \times 50 \mathrm{~mm}, 1,7 \mu \mathrm{m})$ [Waters, Milford, EtatsUnis], à un débit de phase mobile de $0,6 \mathrm{~mL} / \mathrm{min}$, avec les solvants $A($ eau + acide formique à $0,1 \%)$ et $B$ (acétonitrile + acide formique à $0,1 \%)$ utilisés en gradient selon le programme suivant : de $t=0$ à 0,2 minutes $98 \%$ de A et $2 \%$ de $B$, à $t=2$ minutes $2 \%$ de A et $98 \%$ de $B$, à $t=4,7$ minutes $98 \%$ de A et $2 \%$ de $B$. La température de la colonne était fixée à $25^{\circ} \mathrm{C}$ et le volume d'injection de $5 \mu \mathrm{L}$.

Pour la spectrométrie de masse, la tension du capillaire était fixée à $3 \mathrm{kV}$ et le domaine de masses moléculaires analysé de 50 à 1200 Daltons. De la norboldine a également été retrouvée $(\mathrm{m} / \mathrm{z}$ 314,1399 $\pm 2,2$ ppm). Par ailleurs, aucune trace d'ascaridol ou d'eucalyptol n'a été décelée, ce qui est en accord avec le mode d'extraction utilisé, soit l'infusion en eau bouillante. En effet, celleci ne permet pas d'extraire les huiles essentielles, alors que la percolation le permettrait. ${ }^{[1]}$ Au total, dans les extraits infusés, seules la boldine et la norboldine ont été retrouvées au sein d'une liste de molécules recherchées spécifiquement, choisies en raison de leur présence très fortement probable : boldine, norboldine, isoboldine, eucalyptol, ascaridol, linalol, terpinéol. De plus, aucun pic majoritaire n'est apparu en plus de la boldine et de la norboldine.

\section{Discussion}

Dans cette observation, l'éventualité d'une première bouffée délirante dans le cadre d'une pathologie psychiatrique débutante semble peu crédible vu l'âge de la patiente et l'absence de toute récidive avec maintenant plus de 2 ans de recul. L'hypothèse d'une intoxication au cannabis n'a pas été retenue non plus (enfant de 12 ans non pubère qui avait passé toute la journée précédant l'apparition des symptômes chez sa grand-mère).
En faveur de la responsabilité de la consommation d'infusions de feuilles de boldo dans le tableau clinique présenté par l'enfant, on citera la régression spontanée de toute la symptomatologie dès l'interruption de la consommation des tisanes de boldo. L' absence de réapparition des hallucinations ultérieurement va également dans ce sens.

Les indiens Mapuches, communauté aborigène du centre du Chili, utilisent dans leurs rites chamaniques des encens à base de boldo qui sont inhalés par le « machi » (chaman Mapuche). L'essentiel de la culture dans cette communauté étant basée sur la communication avec les esprits de la nature, la sélection du produit utilisé (le boldo) de façon rituelle est importante afin d'obtenir le maximum d'hallucinations visuelles. ${ }^{[3]}$

La boldine, principal alcaloïde du boldo a été mise en évidence en $1872{ }^{[4]}$ En 1999, Bruneton ${ }^{[5]}$ a décrit parmi les alcaloïdes le sous-groupe des aporphinoïdes dont fait partie la boldine, molécule naturelle, mais aussi l'apomorphine, dérivé de synthèse de la morphine. La boldine a des effets diurétiques, sédatifs, tonifiants, antiparasitaires et hépatostimulants. ${ }^{[6]}$ Ces propriétés hépatostimulantes sont à l'origine de l'utilisation en France des infusions de feuilles de boldo. Mais la boldine a aussi des effets neurologiques : analgésiques, hypnotiques, narcotiques, sédatifs, anticonvulsivants et stimulants. ${ }^{[7-9]}$

Chez la souris, Zelter ${ }^{[9]}$ a montré que la boldine injectée en sous-cutané avait une affinité pour les récepteurs D2 dopaminergiques avec des effets neuroleptiques. En 1999, toujours chez la souris, Asencio ${ }^{[10]}$ a confirmé que la boldine injectée par voie péritonéale se fixait sur les récepteurs dopaminergiques D1 et D2 du striatum (action antagoniste avec effet neuroleptique).

Par ailleurs, les feuilles de boldo contiennent 16 autres alcaloïdes que la boldine, dont 5 de type aporphine (boldine comprise) et 3 de type noraporphine dont la norboldine. ${ }^{[11]}$ Elles contiennent aussi 15 huiles essentielles terpéniques notamment l'ascaridol et l'eucalyptol, ${ }^{[12]}$ potentiellement neurotoxiques. ${ }^{[13-15]}$

Pour toutes ces raisons, nous avons posé l'hypothèse que les infusions de feuilles de boldo étaient probablement responsables, chez notre patiente, de la symptomatologie. Mais, afin de mieux préciser ce qui pouvait avoir provoqué les symptômes chez notre patiente, nous avons décidé d'étudier la composition des infusions de sachets de feuilles de boldo commercialisés par la marque Médiflor ${ }^{\circledR}$ et ainsi déterminer que la patiente avait consommé $1,16 \mathrm{mg}$ de boldine tous les soirs pendant 3 jours soit une dose quotidienne de $26 \mu \mathrm{g} / \mathrm{kg}$. Il est néanmoins difficile d'évaluer les conséquences de la consommation d'une telle dose du fait de l'absence de données toxicologiques chez l'Homme, n'ayant que des données chez l'animal avec des effets neuroleptiques de la boldine à des doses de $1,5 \mathrm{mg} / \mathrm{kg}$ en sous cutané. ${ }^{[9]}$

Au final, bien qu'un seul cas humain avec hallucinations ait été signalé après consommation excessive de boldo dans de vieux ouvrages de phytothérapie, ${ }^{[7,16]}$ une intolérance à la boldine reste l'hypothèse la plus probable chez notre patiente, avec peut-être chez elle, une hypersensibilité individuelle particulière à la boldine. 


\section{Conclusion}

L'apparition de troubles du comportement et d'hallucinations sans cause évidente doit faire rechercher à l'interrogatoire la consommation de produits de phytothérapie, notamment d'infusions (« tisanes »). Cette observation renforce l'avis de l'European Medicines Agency qui, en 2009 déconseillait la consommation d'infusion de feuilles de boldo chez l'enfant en raison d'un manque de publications sur le sujet. ${ }^{[17]}$

\section{Conflits d'intérêts. Aucun.}

Abréviations. HPLC-UV : high performance liquid chromatography-ultra violet (chromatographie en phase liquide à haute performance-ultra violet); LIENSs : Littoral Environnement et Société ; UHPLC : ultra high performance liquid chromatography (chromatographie en phase liquide à très haute performance).

\section{Références}

1. Chaboussant PJ. Intoxication aigüe avec troubles du comportement et hallucinations chez une enfant de 12 ans après consommation d'infusions de feuilles de boldo. Thèse pour le Diplôme d'Etat de Docteur en Médecine, Université de Poitiers, 2013

2. Betts TJ. Chromatographic evaluation of boldine and associated alkaloids in boldo. Journal of Chromatography 1990; 511: 373-8 DOI: 10.1016/S00219673(01)93301-1

3. Ratsch C. The Encyclopedia of psychoactive plants, ethnopharmacology and its applications. Rochester, VT: Park Street Press, 2005

4. Bourgoin E, Verne C. Sur l'existence d'un alcali organique dans le boldo. J Pharm Chim 1872; 16: 191-3
5. Bruneton J. Pharmacognosie, phytochimie, Plantes médicinales. Paris : $4^{\text {ème }}$ édition, Lavoisier Tec \& Doc, 2009

6. Steinmetz EF. Codex vegetabilis. Amsterdam, 1957

7. Duke JA. Handbook of medicinal herbs. Boca Raton, FL: $2^{\text {nd }}$ edition, CRC Press, 2002

8. Wichtl M, Anton R. Plantes thérapeutiques. Paris: $2^{\mathrm{è}}$ édition, Lavoisier Tec \& Doc, 2003

9. Zelter G. Neuroleptic-like, anticonvulsivant and antinociceptive effects of aporphine alkaloïds: bulbocapnine, corytuberine, boldine and glaucine. Arch Int Pharmacodyn Ther 1988; 296: 255-81

10. Asencio M, Delaguerrière B, Cassels BK, et al. Biochemical and behavioral effects of boldine and glaucine on dopamine systems. Pharmacol Biochem Behavior 1999; 62: 7-13

11. Petitjean J. Mise à jour sur les propriétés récemment découvertes du boldo, Peumus boldus Molina (monimiacées). Thèse pour le Diplôme d'Etat de Docteur en Pharmacie, Lyon I, 1998

12. Sobecki L. Le boldo (Peumus Boldus) en thérapeutique. Thèse pour le Diplôme d'Etat de Docteur en Pharmacie, Lille II, 1992

13. Bavoux F, Bodiou C, Castot A, et al. Le camphre en pédiatrie, son interêt thérapeutique et ses risques. Therapie 1985; 40: 25-30

14. Gerson M. Camphre et dérivés terpéniques en pédiatrie. Rev Prescrire 1985; 5: $17-20$

15. Bismuth C. Toxicologie clinique. Paris: $5^{\mathrm{è}}$ édition, Flammarion MédecineSciences, 2000

16. Healthcare T. PDR for Herbal Medicines. New-York: 4th Edition, Thomson Reuters, 2007

17. European Medicines Agency. Committee on herbal medicinal products, Assessment on Peumus boldus Molina, Folium, 2009 http://www.ema.europa.eu/docs/en_GB/document_library/Herbal__HMPC_assessment_report/2009/12/WC500018102.pdf Consulté le 23 avril 2014 (15 pages)

Correspondance et offprints : Denis Graber, Service de pédiatrie, Hopital Saint Louis, rue du Dr Schweitzer, 17000 La Rochelle, France

E-mail : denis.graber@ch-larochelle.fr 